\title{
Exercise-induced 31P-NMR metabolic response of human wrist flexor muscles during partial neuromuscular blockade.
}

Quistorff, Bjørn; Mizuno, Masao; Horn, Allan; Secher, Niels H.

Published in:

American Journal of Physiology: Regulatory, Integrative and Comparative Physiology

Publication date:

1994

Document version

Publisher's PDF, also known as Version of record

Citation for published version (APA):

Quistorff, B., Mizuno, M., Horn, A., \& Secher, N. H. (1994). Exercise-induced ${ }^{31}$ P-NMR metabolic response of human wrist flexor muscles during partial neuromuscular blockade. American Journal of Physiology: Regulatory, Integrative and Comparative Physiology, 267, R408-414. 


\title{
Exercise-induced ${ }^{31} \mathrm{P}-\mathrm{NMR}$ metabolic response of human wrist flexor muscles during partial neuromuscular blockade
}

\author{
MASAO MIZUNO, ALLAN HORN, NIELS H. SECHER, AND BJØRN QUISTORFF \\ Copenhagen Muscle Research Center; Nuclear Magnetic Resonance Center, Department of Medical \\ Biochemistry and Genetics, The Panum Institute, DK-2200 Copenhagen N; and Department of \\ Anesthesia, Rigshospitalet, University of Copenhagen, DK-2100 Copenhagen $\emptyset$, Denmark
}

\begin{abstract}
Mizuno, Masao, Allan Horn, Niels H. Secher, and Bjørn Quistorff. Exercise-induced ${ }^{31} \mathrm{P}-\mathrm{NMR}$ metabolic response of human wrist flexor muscles during partial neuromuscular blockade. Am. J. Physiol. 267 (Regulatory Integrative Comp. Physiol. 36): R408-R414, 1994.- The effects of a depolarizing (decamethonium, DECA) and a nondepolarizing neuromuscular blocking agent (vecuronium, VECU) on the phosphorus-31 nuclear magnetic resonance ( $\left.{ }^{31} \mathrm{P}-\mathrm{NMR}\right)$-detected metabolic response to muscle contractions were studied separately in six healthy untrained males. Subjects who showed splitting of the $P_{i}$ peak during graded rhythmic forearm exercise without the drugs were selected. It was found that both drugs abolished the $P_{i}$ peak splitting during exercise. Despite a similar reduction in phosphocreatine (PCr) during exercise with each drug, a smaller increase in $P_{i}$ was observed with DECA than with VECU $(P<0.05)$. End-exercise muscle $\mathrm{pH}$ was higher with DECA $(6.93 \pm 0.07)$ than with VECU $(6.79 \pm 0.11)(P<0.05)$. The $\mathrm{PCr}$ and $\mathrm{P}_{\mathrm{i}}$ recovery was twoand threefold faster with DECA than with VECU, respectively $(P<0.05)$. On the basis of the concept that depolarizing and nondepolarizing agents have a preferential effect on fast- and slow-twitch muscle fibers, respectively, the present results support the hypothesis that the NMR-observed splitting of the $P_{i}$ peak reflects the metabolic differences between the two major fiber types of human skeletal muscle.
\end{abstract}

decaniethonium; muscle fiber types; forearm exercise; inorganic phosphate; muscle $\mathrm{pH}$; phosphocreatine; vecuronium

NONDEPOLARIZING and depolarizing neuromuscular blocking agents affect preferentially red and white skeletal muscle, respectively, as demonstrated among various muscles in several animal species (11, 21, 32). Human skeletal muscle is heterogeneous with respect to muscle fiber types, often classified as slow- (ST; type I or oxidative) and fast-twitch (FT; type II or glycolytic) fibers $(15,22)$. Typically, an equal proportion of the two major fiber types is present, although a large variation exists among muscles and subjects (22). However, the preference of the two types of neuromuscular blocking agents also appears to be valid for human skeletal muscle. The nondepolarizing agent tubocurarine affects a head lift more than a handgrip (12), whereas the opposite is true for the depolarizing agent decamethonium (DECA) (13). Within a single muscle group, a preference of the two types of neuromuscular blocking agents for a specific fiber type is indicated by an opposite effect on fatigue $(14,18)$. Furthermore, DECA affects rate of rise in tension more than endurance, whereas the reversed effect is seen with tubocurarine $(23,25,27)$, as also reflected in the electromyographic response $(14,23)$.

An alternative approach has been made by histochemical evaluation of muscle-biopsy samples. A correlation is demonstrated between the degree of neuromuscular blockade by tubocurarine and the relative occurrence of ST fibers in the soleus, gastrocnemius, and vastus lateralis muscles (26). On the basis of the glycogen depletion pattern, ST fibers are affected to a greater extent than FT fibers in thigh muscles during cycling with tubocurarine (3). In contrast, the depolarizing agent suxamethonium has a preferential effect on glycogen depletion of FT fibers (24). Thus energy metabolism during exercise under partial neuromuscular blockade depends on which type of muscle fiber remains relatively unaffected.

The use of phosphorus-31 nuclear magnetic resonance ( ${ }^{31} \mathrm{P}-\mathrm{NMR}$ ) spectroscopy allows continuous monitoring of relative changes in intramuscular concentrations of several metabolites, including $\mathrm{P}_{\mathrm{i}}$, phosphocreatine ( $\mathrm{PCr}$ ), and ATP. A noninvasive determination of intracellular $\mathrm{pH}$ in human skeletal muscle can be performed as well. A wide variation exists even among untrained individuals with respect to response pattern of these energy metabolites during forearm exercise and recovery $(2,17$, 20). This metabolic variation has been related to contractile and metabolic characteristics of specific fiber types $(1,15,17,20,31)$ rather than to different proportion of active and inactive fibers and/or muscle groups within the wrist and finger flexors $(7,8,10,30)$. Experiments combining ${ }^{31} \mathrm{P}-\mathrm{NMR}$ and muscle-biopsy technique have shown that muscle $\mathrm{pH}$ compartmentation in the form of splitting of the $\mathrm{P}_{\mathrm{i}}$ peak, differentiating high- and low-pH $P_{i}$ peaks during exercise observed in some individuals (1, $20,31)$, reflects the metabolic characteristics of ST and FT fibers (17). Furthermore, an abolished splitting of the $\mathrm{P}_{i}$ peak has been reported during forearm rhythmic exercise with partial curarization (16).

The aim of this study was to evaluate the hypothesis that the NMR-observed splitting of the $\mathrm{P}_{\mathrm{i}}$ peak during rhythmic forearm exercise reflects the metabolic differences between the two major fiber types of skeletal muscle. Inasmucl as the preferential effects of the two types of neuromuscular blocking agents on ST and FT fibers liave been well established, the above hypothesis predicts that administration of the depolarizing agent DECA suppresses primarily the low-pH component of the $\mathrm{P}_{\mathrm{i}}$ peak during exercise, whereas the nondepolarizing agent vecuronium (VECU) mainly affects the high-pH $\mathrm{P}_{\mathrm{i}}$ peak,

\section{METHODS}

Subjects. The nondominant arm of six healthy untrained males (age 21-25 yr, weight $60-70 \mathrm{~kg}$, height $175-183 \mathrm{~cm}$ ) was studjed. Subjects were fully informed about the nature and possible risks of the experiments before they gave written 
consent to participate in the study, which was approved by the Local Ethics Committee.

All subjects had participated in previous NMR experiments (17) and herein demonstrated muscle pH compartmentation in the form of high- and low-pH $\mathrm{P}_{\text {; }}$ peaks during graded rhythmic forearm exercise. Forearm circumference, maximal voluntary contraction force (MVC), and maximal rhythinic exercise capacity were $25.5 \pm 1.1(\mathrm{SD}) \mathrm{cm}, 27.0 \pm 3.3 \mathrm{~N} \cdot \mathrm{m}$, and $1.6 \pm 0.3 \mathrm{~W}$, respectively. Four subjects participated in the muscle-biopsy procedure and showed an even proportion of the two major fiber types in the flexor carpi radialis muscle $(47 \pm$ $5 \% \mathrm{ST}, 24 \pm 14 \% \mathrm{FT}_{\mathrm{a}}$, and $29 \pm 17 \% \mathrm{FT}_{\mathrm{b}}$ fibers) (17).

NMR spectroscopy. A spectrometer (Vivospec, Otsuka Electronics) and an 80-cm-long 2.9-T horizontal superconducting magnet with a $26-\mathrm{cm}$ bore (Magnex Scientific) were used. A 4-cm-diam two-turn surface coil was placed over the wrist flexor muscles centered at a point corresponding to one-fourth the distance between the olecranon and the styloid process. Thus the coil was expected to cover the flexor carpi radialis muscie and also portions of the palmaris longus and the flexor carpi ulnaris, as well as the flexor digitorum superficialis and the flexor digitorum profundus muscle.

Homogeneity of the magnetic field was optimized by shimming on the proton signal. Line width before experiments was $<0.5$ parts per milhion (ppm). ${ }^{31} \mathrm{P}$ spectra were obtained at $49.83 \mathrm{MHz}$ by single $90^{\circ}$ pulse excitations with pulse-power and -width optimized by the height of the PCr peak. Data were collected in $2 \mathrm{~K}$ points over $205 \mathrm{~ms}$ at $6 \mathrm{~s}$ of interpulse delay. Data acquisition was performed for $1.5 \mathrm{~min}$ at rest, during exercise, and continuously in blocks of $30 \mathrm{~s}$ at the end of exercise and during the first $2 \mathrm{~min}$ of recovery, followed by blacks of 1 min during the remaining $13 \mathrm{~min}$ of recovery (Fig. 1). In ${ }^{31 p}$ spectra (16 scans) obtained at rest, the signal-to-noise ratio ranged from 12 to 16 .

Spectral analysis. All spectra were analyzed by the same operator without knowledge of the type of experiments. After application of exponential line broadening of $5 \mathrm{~Hz}$ to each spectrum, muscle $\mathrm{pH}$ was calculated according to Taylor et al. (30). The chemical shift value at the maximum height(s) of $P_{i}$ resonance(s) was used for this calculation.

The peak area of $P_{i}, P C r$, and $\beta$-ATP was measured by a curve-fitting program of the Vivospec spectrometer software with assumption of Lorentzian line shape. Baseline correction was performed semi-automatically by manual choosing of eight points at the same chemical shifts in all spectra. The program subsequently identified the peaks of phosphomonoester (PME), $P_{i}, P C r$, and $A^{\prime} T P$. When splitting of the $P_{i}$ peak was not identified by the program, the signal was considered as a single peak even when the $P_{\text {i }}$ peak was broadened.

The peak areas were corrected for partial saturation. Saturation factors, determined as the ratio of the peak area in spectra obtained with 6- and 30-s interpulse delays, were 1.44 for $\mathrm{P}_{i}$, 1.41 for $\mathrm{PCr}$, and 1.15 for $\beta$-ATP. During the course of the experiments, the total ${ }^{31} \mathrm{P}$ signal $\left(\mathrm{PME}+\mathrm{P}_{i}+\mathrm{PCr}+\beta\right.$-ATP) was constant within $10 \%$. The coefficient of variation in double determinations of the peak area was $1.6 \%$ for high-pH $\mathrm{P}_{\mathrm{i}}, 1.3 \%$ for low-pH $\mathrm{P}_{i}, 2.8 \%$ for $\mathrm{PCr}$, and $1.8 \%$ for $\beta$-ATP. In all spectra the total $\mathrm{P}_{\mathrm{i}}$ peak area was used for calculating the $\mathrm{P}_{\mathrm{i}}$-to- $\mathrm{PCr}$ ratio $\left(\mathrm{P}_{i} / \mathrm{PCr}\right)$. The area of $\mathrm{PCr}$ and $\beta$-ATP was expressed as percentage of the resting value, and fold change was used for $\mathrm{P}_{i}$. The kinetics of $\mathrm{PCr}$ resynthesis and $\mathrm{P}_{\mathrm{i}}$ decrease during recovery was evaluated by fitting data to a monoexponential function with application of least-square regression. The half time $\left(t_{1 / 2}\right)$ of recovery was calculated from the fitted curve.

Exercise protocol. The nonmagnetic forearm ergometer and the protocol for determination of maximal intensity of forearm rhythmic exercise (one contraction every 6 s) have been described (17). The subject lay face down on a stretcher with the nondominant arm in the magnet. The elbow joint was slightly bent, and the flexor carpi radialis muscle was centered over the surface coil. Before the drug experiments, graded forearm exercise was conducted with $40 \%$ maximal intensity for $3 \mathrm{~min}$ followed by $80 \%$ for $2 \mathrm{~min}$ (Fig. 1). After $30 \mathrm{~min}$ of recovery, the same absolute intensity was repeated for $4 \mathrm{~min}$ without neuromuscular blockade (control) and then with either DECA ar VECU, followed by $15 \mathrm{~min}$ of recovery. Three experiments on the same subject were performed, with $\geq 1$ wk interyal between them.

To quantitate the reduction in muscle strength caused by the neuromuscular blockade, MVC was determined in the same arm ergometer attached inside the magnet at the end of $30 \mathrm{~min}$ of recovery after graded exercise. Thereafter, a bolus containing $0.01 \mathrm{mg}$ VECU (Organon) or $0.02 \mathrm{mg}$ DECA (Burroughs-Wellcome) per kilogram body weight, followed by $20 \mathrm{ml}$ saline, was administered intravenously through a catheter placed in a vein of the nonexercising arm. Small supplementary doses were given at 3- to 5-min intervals as required until the $50 \%$ MVC level was reached. It has been well documented that the effect of partial neuromuscular blockade imposed by either type of agent lasts $\geq 5 \mathrm{~min}$, as evaluated by repeated measurements of MVC in limb skeletal muscle $(3,12$, $13,18,25,27)$. In all experiments an Ambu-E resuscitator apparatus, neostigmine, and atropine were available but never needed.

\section{Graded exercise}

\section{Drug}

AMR
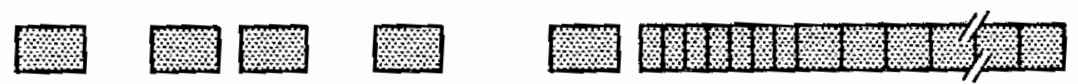

Wintensity
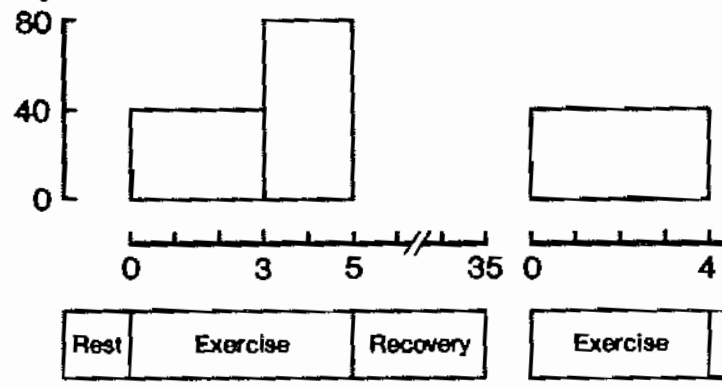

0

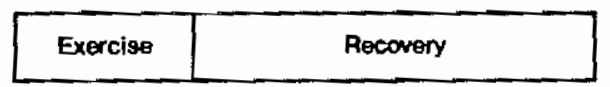

Fig. 1. Schematic experimental protocol Three series of experiments were performed consisting of graded forearm exercise at 2 intensities and repeated exercise with $40 \%$ intensity without (CONT) or with partial neuromuscular blockade imposed by either decamethonium (DECA) or vecuronium (VECU). 
Statistics. Values are expressed as means \pm SD. Comparisons between related observations and between independent samples were performed by Friedman's two-way analysis of variance by rank and by the Kruskal-Wallis one-way analysis of variance by rank, respectively (28). When significant differences were detected, these were located by the Wilcoxon's matched-pairs signed-rank test or the Mann-Whitney $U$ test. A $5 \%$ level was considered significant for two-tailed tests.

\section{RESULTS}

Control. Metabolic response to graded forearm exercise did not differ on three separate days (Figs. 2 and 3 ). The mean values of $\mathrm{P}_{\mathrm{i}} / \mathrm{PCr}$ and muscle $\mathrm{pH}$ at rest were $0.11 \pm 0.02$ and $7.03 \pm 0.04$, respectively. A line width of $18 \pm 4 \mathrm{~Hz}$ was observed in the $\mathrm{P}_{i}$ peak at rest. During $40 \%$ maximal intensity, two components of the $\mathrm{P}_{i}$ peak were evident, differentiating a high-pH and a low-pH peak $(P<0.05)$, At this exercise intensity the high-pH $P_{\text {; }}$ was larger than the low-pH $P_{i}(P<0.05)$ and remained constant throughout exercise, whereas the low-pH $P_{\mathrm{i}}$ further increased at $80 \%$ intensity $(P<0.05)$. Similarly, the line width of the high $-\mathrm{pH} \mathrm{P}_{\text {, peak }}, 30 \pm 3$ $\mathrm{Hz}$, was larger than that of the low $-\mathrm{pH} \mathrm{P}$, peal, $18 \pm 3$ $\mathrm{Hz}$, at $40 \%$ exercise intensity $\left(P^{*}<0.05\right) . \mathrm{At} 80 \%$ intensity, the line width of the high-pH $\mathrm{P}_{3}$ peak remained unchanged $(31 \pm 8 \mathrm{~Hz})$, whereas that of the low-pH $P_{\text {i }}$ peak further increased $(23 \pm 3 \mathrm{~Hz})(P<0.05)$ but was still lower than the value for the high-pH $\mathrm{P}_{\mathrm{i}}$ peak $(P<0.05)$. There was a difference in end-exercise muscle $\mathrm{pH}$ between the two components $(0.48 \pm 0.12)$. During 40 and $80 \%$ exercise intensity, $\mathrm{PCr}$ decreased to $48.2 \pm 6.9$ and $44.8 \pm 6.3 \%$ of the resting value, respectively $(P<0.05)$. The ATP also decreased slightly, to $92 \pm 5 \%$ during $40 \%$ intensity and to $86 \pm 5 \%$ of the resting value during $80 \%$ intensity $(P<0.05)$.

At the end of 30 min of recovery after graded exercise, $\mathrm{P}_{\mathrm{j}} / \mathrm{PCr}$ and muscle $\mathrm{pH}$ had returned to the resting levels
Fig. 2. Muscle $\mathrm{pH}$ at rest, during graded forearm exercise with 40 and $80 \%$ intensity, and during repeated exercise with same absolute intensity (40\%) followed by 15 min of recovery without or with partial neuromuscular blockade imposed by either decamethonium or vecuronium. Open symbols, low-pH $P_{i}$; closed symbols, high-pH $P_{1}$. Only a single $P_{i}$ peak was evident during exercise with blocking agents. An estimated preferential effect of each blocking agent is noted. Values are means $\pm \operatorname{SD}(n=6)$.

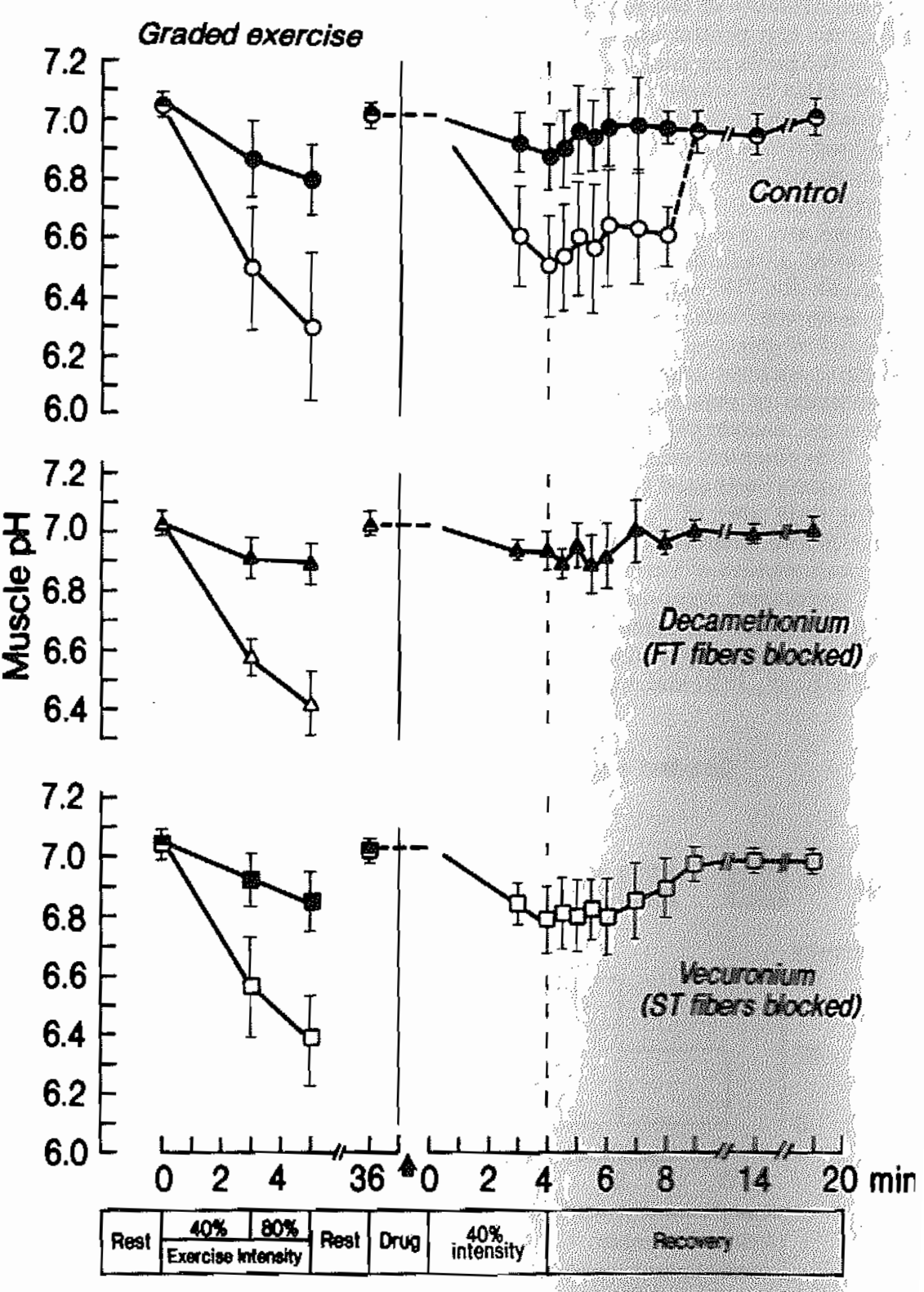




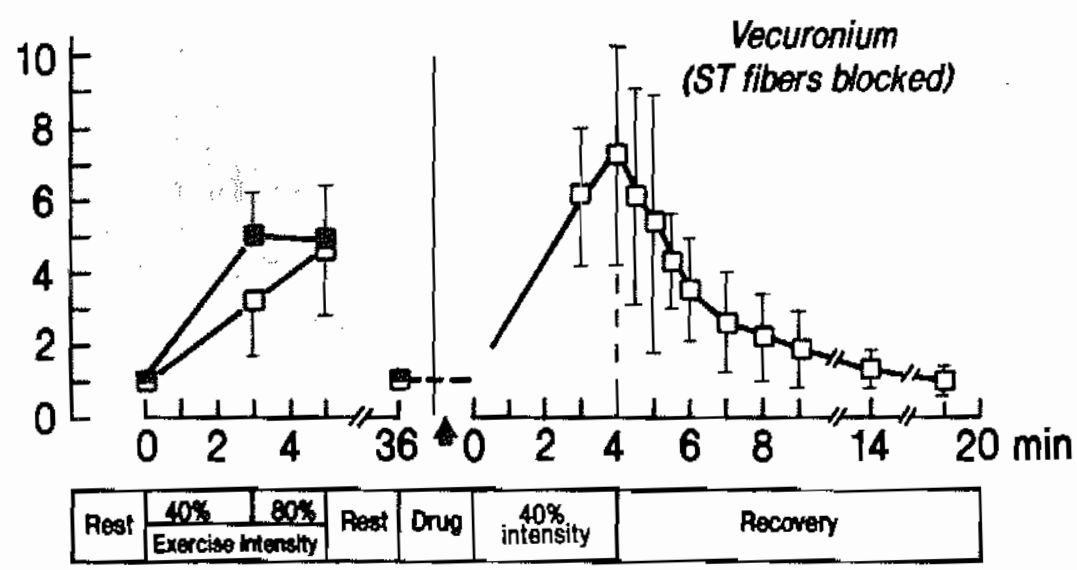

Fig. 3. Fold changes in $P_{i}$ peak relative to individual resting values during control exercise with 40 and $80 \%$ intensity and repeated exercise with same absolute intensity (40\%) followed by $15 \mathrm{~min}$ of recovery without or with partial neuromuscular blockade imposed by either decamethonium or vecuronium. (See legend to Fig. 2).

(Table 1, Fig. 2). When the subjects subsequently performed forearm exercise with $40 \%$ maximal intensity in the control experiments, splitting of the $P_{i}$ peak was again distinct (Figs. 2-4). The line width of the high-pH $\mathrm{P}_{i}$ peak was $31 \pm 7 \mathrm{~Hz}$, larger than that of the low-pH $\mathrm{P}_{i}$ peak, $19 \pm 7 \mathrm{~Hz}$. Neither the muscle $\mathrm{pH}$ nor $\mathrm{P}_{\mathrm{i}}$ peak response in the two components was significantly differ- ent from those obtained at $40 \%$ intensity during graded exercise. PCr and ATP decreased by $48.5 \pm 6.9$ and $87.2 \pm 6.4 \%$ of resting values, respectively $(P<0.05)$, which was the same decrease as that observed at $40 \%$ intensity during graded exercise. The $t_{1 / 2}$ of recovery for the high-pH $\mathrm{P}_{\mathrm{i}}$ peak was twice as fast as that for the low$\mathrm{pH} \mathrm{P}_{\mathrm{i}}$ peak $(P<0.05$; Table 1$)$. After $15 \mathrm{~min}$ of recovery,

Table 1. PCr and $P_{i} / P C r$ at rest, during rhythmic exercise with $40 \%$ intensity, and after 15 min of recovery as well as half-time of recovery for $P_{i}$ and $P C r$ determined in control, decamethonium, and vecuronium experiments

\begin{tabular}{|c|c|c|c|c|c|c|c|c|c|}
\hline & \multicolumn{3}{|c|}{$\mathrm{PCr} \%$} & \multicolumn{3}{|c|}{$\mathrm{P}_{\mathrm{i}} / \mathrm{PCr}$} & \multicolumn{3}{|c|}{ Half-Tìme of Recovery, min } \\
\hline & Rest & Exercise & Recovery & Rest & Exercise & Recovery & High-pH $P_{i}$ & Low-pH $P_{1}$ & $\mathrm{PCr}$ \\
\hline 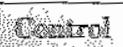 & 100 & $48.5 \pm 6.9$ & $94.4 \pm 5.6$ & $0.11 \pm 0.03$ & $1.69 \pm 0.54$ & $0.11 \pm 0.03$ & $0.35 \pm 0.12$ & $1.18 \pm 0.46$ & $1.29 \pm 0.53$ \\
\hline 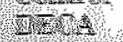 & 100 & $54.7 \pm 7.8$ & $98.5 \pm 3.4$ & $0.11 \pm 0.02$ & $1.00 \pm 0.24^{*}$ & $0.09 \pm 0.03$ & 0.52 & 0.15 & $0.82 \pm 0.32$ \\
\hline 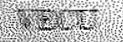 & 19.9. & $51.5 \pm 11.2$ & $92.7 \pm 3.2 \div$ & $0.10 \pm 0.02$ & $1.50 \pm 0.49 \dagger$ & $0.10 \pm 0.05$ & 1.39 & $0.74 \dagger$ & $1.50 \pm 0.48$ \\
\hline
\end{tabular}

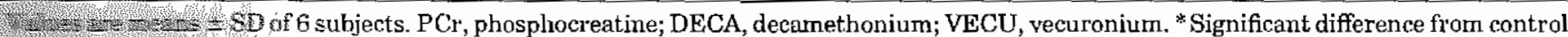

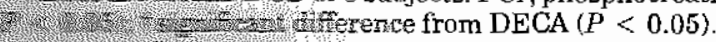


Fig. 4. Phosphorus-31 nuclear magnetic resonance ( $\left.{ }^{31} \mathrm{P}-\mathrm{NMR}\right)$ speetra obtained rest and duriug forearm exercise with $40 \%$ intensity without and with partial neuromuscular blockade imposed by either decamethonium or vecuronium. peak. Exponential line broadening of 5 $\mathrm{Hz}$ was used for each spectrum (the sum of 16 scans). from wrist flexor muscles in 1 subj at Both agents prevented splitting of the $P_{i}$

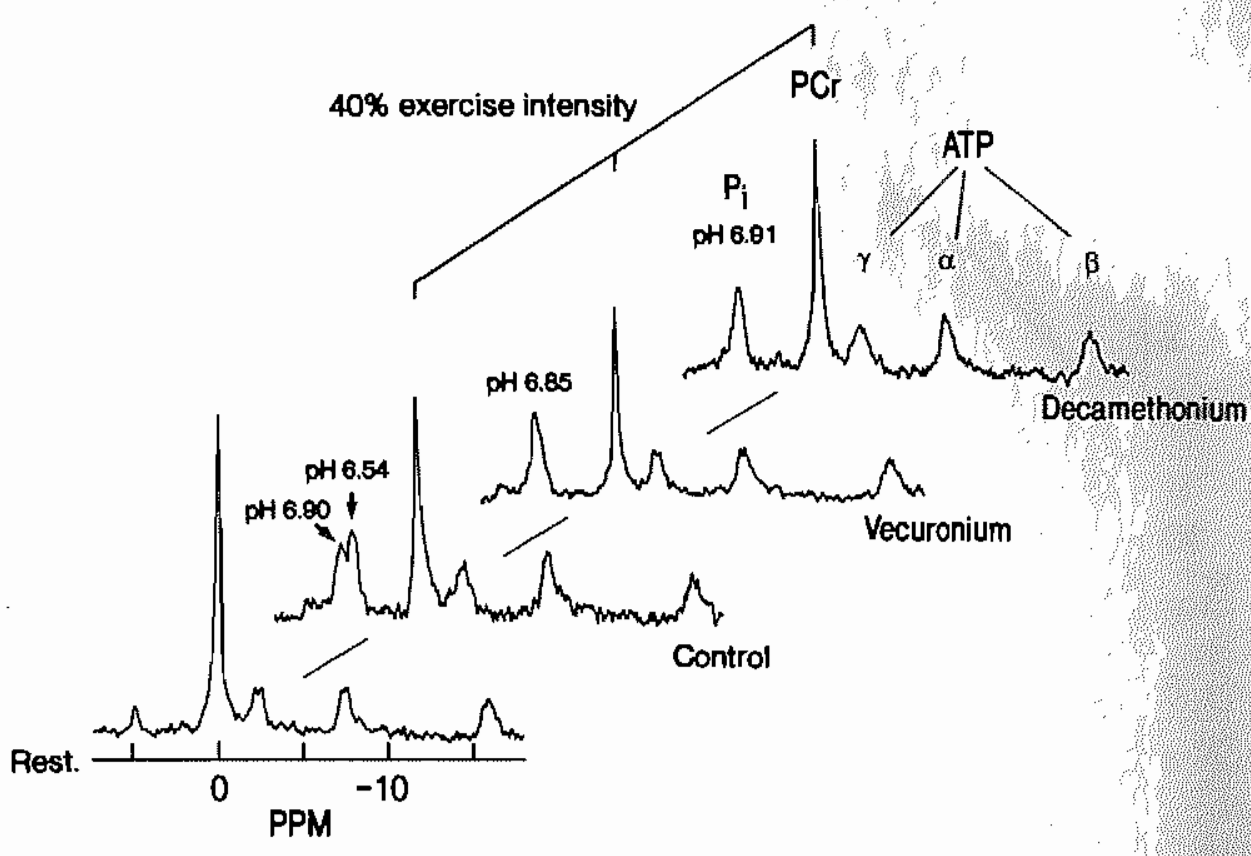

Muscle $\mathrm{pH}$ returned to the resting value after 3 min of muscle $\mathrm{pH}$ and $\mathrm{P}_{\mathrm{i}}$ returned to resting values, whereas PCr showed a slightly lower value, $94.4 \pm 5.6 \%$ of the resting value $(P<0.05)$.

Effect of neuromuscular blockade. Administration of $1.7 \pm 0.3 \mathrm{mg}$ DECA or $1.0 \pm 0.02 \mathrm{mg}$ VECU reduced forearm MVC to $52 \pm 3$ and $51 \pm 3 \%$ of control values, respectively. No difference was found in the time necessary to establish this force reduction between DECA, $10 \pm 5 \mathrm{~min}$, and VECU, $10 \pm 2 \mathrm{~min}$. During rhythmic forearm exercise with the same absolute intensity, no splitting of the $P_{i}$ peak was observed with either DECA or VECU (Figs. 2-4). End-exercise muscle $\mathrm{pH}$ with DECA was $6.93 \pm 0.07$, slightly but significantly higher than with VECU, $6.79 \pm 0.11(P<0.05)$. Despite a similar reduction in $\mathrm{PCr}$ during exercise between the two neuromuscular blocking agents, a smaller increase in $P_{i}$ was observed with DECA than with VECU $(P<$ 0.05 ; Table 1, Fig. 3). Thus $\mathrm{P}_{\mathrm{i}} / \mathrm{PCr}$ during exercise was significantly lower with DECA than with VECU $(P<$ 0.05 ). The line width of the $P_{i}$ peak during exercise was similar between DECA, $34 \pm 7 \mathrm{~Hz}$, and VECU, $35 \pm 3$ $\mathrm{Hz}$. At the end of exercise ATP was $90 \pm 6 \%$ of the resting value with DECA and $91 \pm 5 \%$ with VECU.

Compared with the control data, end-exercise muscle $\mathrm{pH}$ with DECA was similar to the high-pH $\mathrm{P}_{\mathrm{i}}$ peak, whereas the value with VECU was lower than the high-pH but higher than the low-pH $P_{i}$ peak $(P<0.05$; Fig. 2). Because the highest increase in $P_{i}$ was observed during exercise with VECU (Fig. 3), $\mathrm{P}_{\mathrm{i}} / \mathrm{PCr}$ during exercise was lower with DECA than in the control experiment $(P<0.05)$, but no difference was demonstrated between VECU and the control experiments (Table 1). The line width of the $P_{i}$ peak during exercise with DECA and VECU was higher than that of the low-pH $\mathrm{P}_{\mathrm{i}}$ peak $(P<0.05)$ but similar to that of the high-pH $\mathrm{P}_{\mathrm{i}}$ peak observed in the control experiments. The reduction in $\mathrm{PCr}$ and ATP during exercise with DECA or VECU did not differ significantly from the control value. 2). The $t_{1 / 2}$ of recovery for $\mathrm{PCr}$ and $\mathrm{P}_{\mathrm{i}}$ after exercise was two- and threefold faster with DECA than with VECU $(P<0.05$; Table 1$)$. At $15 \mathrm{~min}$ of recovery after exerise with each agent, muscle $\mathrm{pH}$ and $\mathrm{P}_{\mathrm{i}}$ had returned to resting values, whereas a slightly higher value of PC. was observed with DECA than with VECU $(P<0.05)$ ATP returned to the resting level by $1 \mathrm{~min}$ of recovery. with no difference between DECA $(101 \pm 7 \%)$ and VECU $(104 \pm 8 \%)$.

Compared with the control values, the $t_{1 / 2}$ of recovers for $P_{i}$ after exercise with DECA was similar to the value obtained in the high-pH $P_{i}$ peak, and the value with VECU corresponded to that observed in the low-pH $\mathrm{P}$ peak (Table 1). The $t_{1 / 2}$ for $\mathrm{PCr}$ after exercise with DECA was faster than the control value $(P<0.05$. whereas no difference was found in the $t_{1 / 2}$ between VECU and the control experiments.

\section{DISCUSSION}

NMR response. In the present study, it was hypothesized that the NMR-detected splitting of the $P$ poet during exercise reflects the metabolic differences be tween ST and FT fibers in working muscles, as propeses previously (20). This hypothesis was tested by avplture the two types of neuromuscular blocking agents 40 impose partial neuromuscular blockade during exere:-s The depolarizing agent DECA is predicted to afteti primarily FT fibers, and consequently the N.rS tesponse during exercise will reflect mainly metat tcharacteristics of ST fibers possessing relatively th oxidative potential. Conversely, VECU is expected to block selectively ST fibers, and thus the NMR resectuse will be a reflection of unaffected FT fibers with het glycolytic capacity. The present NMR data demure-trating an abolishment of $\mathrm{P}_{\mathrm{i}}$ peak splitting durire partial recovery with DECA and after 5 min with VECU (Fis: .61), esulte igh-p ion ar gnific xperir. pite $\mathrm{t}$ 
neuromuscular blockade induced by each drug as well as different dynamics in muscle $\mathrm{pH}, \mathrm{P}_{\mathrm{i}}$, and $\mathrm{PCr}$ during rhythmic forearm exercise and lecovery between the two drugs support the hypothesis.

Muscle $\mathrm{pH}$ compartmentation in the form of $\mathrm{P}_{\mathrm{i}}$ peak splitting during rhythmic forearm exercise is associated with an even proportion of two major fiber types in working muscles (17). As observed during graded exercise in the present subjects, the kinetics of the high- and the low-pH $\mathrm{P}_{\mathrm{i}}$ peak during and after exercise corresponds well to the metabolic characteristics of ST and FT fibers, respectively $(1,15,17,20,31)$. When the subjects subsequently repeated rhythmic forearm exerxise during partial neuromuscular blockade, only a single $P_{i}$ peak was seen (Fig. 4).

Although muscle force was reduced to the same extent roy $\sim 50 \%$ MVC) by each agent, metabolic differences (retween DECA and VECU were obtained during and tater rhythmic forearm exercise. Exercise-induced acidosis in the wrist flexor muscles was slightly but signifisently less pronounced with DECA than with VECU YYig. 2). Both $\mathrm{PCr}$ and $\mathrm{P}_{\mathrm{i}}$ showed a faster recovery after Exercise with DECA than with VECU (Fig. 3). The $t_{1 / 2}$ xecovery values of $\mathrm{P}_{\mathrm{i}}$ after exercise with DECA and TECU correspond well to those of the high- and the (6w-pH $\mathrm{P}_{\mathrm{i}}$ peak values obtained without partial neurosisuscular blockade, respectively (Table 1). Furthermore, (3ese NMR-detected differences in muscle energetics istween the two types of neuromuscular blocking agents 3rrespond to those previously described for subjects Sith a predominance of either ST or FT fibers in the Wist flexor muscles (17). It is noteworthy that the isperimental protocol of this study was aimed at evalu-ing the reproducibility of NMR data obtained on three parate days. No significant difference was found in tzabolic response to graded rhythmic forearm exercise t

Selectivity of neuromuscular blocking agents. On the is of NMR-detected metabolic response to exercise VECU, a degree of selectivity of the nondepolarizzagent VECU for ST fibers may be questioned. The : width of the single $P_{i}$ peak during exercise with CU was expected to be larger than the value with 0A, because 1.6-fold larger line width was found in high- than the low-pH $\mathrm{P}_{\mathrm{i}}$ peak during control rise without neuromuscular blockade (Fig. 4). reas the line width obtained with DECA was similar vat of the high-pH $P_{\text {i }}$ peak in the control, the value VECU was larger than that of the low-pH $P_{i}$ peak was similar to the value with DECA. Similarly, exercise muscle $\mathrm{pH}$ with VECU $(\mathrm{pH}$ 6.79) was er than that of low-pH $\mathrm{P}_{\mathrm{i}}$ at $40 \%$ intensity during ad exercise without neuromuscular blockade $(\mathrm{pH}$ whereas rhythmic forearm exercise with DECA in a similar level of acidosis observed in the ${ }_{\mathrm{pH}} \mathrm{P}_{\mathrm{i}}$ ( $\mathrm{pH}$ 6.93; Fig. 2). Furthermore, the reducand resynthesis of $\mathrm{PCr}$ with VECU did not differ iteantly from those with DECA or the control timent (Table 1). These results were obtained dethe fact that subjects performed at the same absolute exercise intensity both with and without partial neuromuscular blockade.

In the study in which the glycogen depletion pattern in the two major fiber types of thigh muscles was evaluated during cycling followed by administration of the nondepolarizing agent tubocurarine, $\sim 60 \%$ of ST and $40 \%$ of $\mathrm{FT}$ fibers were estimated to be affected (3). Compared with this selectivity of tubocurarine to $\mathrm{S}^{\prime} \mathrm{T}$ fibers, the depolarizing agent suxamethonium shows a relatively higher degree of preference to FT fibers, i.e., $\sim 90 \%$ of FT fibers and $30 \%$ of ST fibel's are estimated to be affected on the basis of the glycogen depletion pattern (24). Thus NMR-detected metabolic response with VECU may indicate that ST and FT fibers were blocked to a similar extent. This was not, however, reflected in tle chemical shift of $P_{i}$ pools, because a single $P_{i}$ peak was observed throughout exercise with VECU. Alternatively, the nondepolarizing agent VECU may have an inhibitory effect on glycolysis.

Mechanism of preferential effects. What is the mechanism underlying the preferential effects of nondepolarizing and depolarizing neuromuscular agents on skeletal muscle? One explanation could be sought in a relationship between morphological as well as electrophysiological characteristics of motor end plates and the amount of a blocking agent reaching a neuromuscular junction. In animal skeletal muscle, ample evidence is available demonstrating that the size of motor end plates is two to three times smaller in ST than in FT muscles $(4,5,19$, 29). Furthermore, ST muscles also show two- to threefold lower activity of acetylcholinesterase and a different distribution of its molecular forms compared with FT muscles (9). In humans a large variation in acetylcholine content at neuromuscular junctions of intercostal muscles has been observed after nerve stimulation (6). With the assumption that $\mathrm{S}^{\prime} \mathrm{T}$ fibers receive the smallest amount of acetylcholine, the competitive blockade developed by a nondepolarizing neuromuscular blocking agent would cause a more pronounced inhibition primarily in these fibers. On the other hand, the smallest rise in acetylcholine concentration may be necessary for $\mathrm{F}^{\prime} \mathrm{T}$ fiber's to be depolarized, and thus these fibers are the first to be blocked by acetylcholine-type (depolarizing) neuromuscular blocking agents.

In conclusion, the results demonstrate a substantial difference in muscle $\mathrm{pH}, \mathrm{P}_{\mathrm{i}}$, and $\mathrm{PCr}$ response during and after contractions from partial neuromuscular blockade between those imposed by VECU and those by DECA. On the basis of the concept that the nondepolarizing and depolarizing neuromuscular agents have a preferential effect on ST and FT fibers, respectively, the present results support the hypothesis that the NMRobserved splitting of the $\mathrm{P}_{\mathrm{i}}$ peak reflects the two major fiber types of skeletal muscle.

This study was supported by grants from the Danish National Research Foundation (nos. 5.1.7.5.2.14, 12-9789, and 12-9793 to B. Quistorff), the Direktør E. C. IIertz Fund (to B. Quistorff). and the Direktør J. and H. O. Madsens Fund (to M. Mizuno)

Address for reprint requests: M. Mizuno, NMR Center, Depl. of Medical Biochemistry and Genetics, The Panum Inslitute, L"niv. of Copenhagen, Blegdamsvej 3, DK-2200 Copenhagen N, Denınark.

Received 16 Augnst 1993; accepted in final form 23 Fehruary 1994 


\section{REFERENCES}

1. Achten, E., M. Van Cauteren, R. Willem, R. Luypaert, W. J. Malaisse, G. Van Bosch, G. Delanghe, K. De Meirleir, and M. Osteaux. ${ }^{31} \mathrm{P}-\mathrm{NMR}$ spectroscopy and the metabolic properties of different muscle fibers, J. Appl. Physiol. 68: 644-649, 1990.

2. Bendahan, D., S. Confort-Gouny, G. Kozak-Reiss, and P. J. Cozzone. Heterogeneity of metabolic response to muscular exercise in humans. New criteria of invariance defined by in vivo phosphorus-31 NMR spectroscopy. FEBS Lett. 272: 155-158, 1990.

3. Bonde-Petersen, F., P. D. Gollnick, 'T'. I. Hansen, N. Hultén, J. H. Kristensen, N. Secher, and O. Secher. Glycogen depletion pattern in human muscle fiber during work under curarization (d-tubocurarine). In: Metabolic Adaptation to Prolonged Physical Exercise, edited by H. Howald and J. R. Poortmans. Basel: Birkhäuser Verlag, 1975, p. 430-442.

4. Duchen, L. W. An electron microscopic comparison of motor end-plates of slow and fast skeletal muscle fibres of the mouse. $J$. Neurol. Sci, 14: 37-45, 1971.

5. Ellisman, M. H., J. E. Rash, L. A. Staehelin, and K. R. Porter. Studies of excitable membranes. II. A comparison of specialization at neuromuscular junctions and nonjunctional sarcolemmas of mammalian fast and slow twitch muscle fibers. $J$. Cell Biol. 68: 752-774, 1976

6. Elmqvist, D., and D. M. J. Quastel. A quantitative study of end-plate potentials in isolated human muscle. J. Physiol. Lond. 178: 505-529, 1965

7. Fleekenstein, J. L., L. A. Bertocei, R. L. Nunnally, R. W. Parkey, and R. M. Peshoek. Exereise-enhanced MR imaging of variations in forearm muscle anatomy and use: importance in $\mathrm{MR}$ spectroscopy. Am. J. Radiol. 153: 693-698, 1989.

8. Fleckenstein, J. L., D. Watumull, L. A. Bertocci, R: w. Parkey, and R. M. Peshock. Finger-specific flexor recruitment in humans: depiction by exercise-enhanced MRI. J. Appl. Physiol. 72: 1974-1977, 1992.

9. Gisiger, V., and H. R. Stephens. Asymmetric and globular forms of AChE in slow and fast muscles of 129/ReJ normal and dystrophic mice. J. Neurochem. 41:919-929, 1983.

10. Jeneson, J. A. L., J. S. Taylor, D. B. Vigneron, T. S. Willard, L. Carvajal, S. J. Nelson, J. Murphy-Boesch, and T. R. Brown. ${ }^{1} \mathrm{H}$ MR imaging of anatomical compartments within the finger flexor muscles of the human forearm. Magn. Reson. Med. 15: 491-496, 1990.

11. Jewell, P. A., and E. Zaimis. A differentiation between red and white muscle in the cat based on responses to neuromuscular blocking agents. J. Physiol. Lond. 124: 417-428, 1954.

12. Johansen, S. H., M. Jørgensen, and S. Molbech. Effect of tubocurarine on respiratory and nonrespiratory muscle power in man. J. Appl. Physiol. 19: 990-994, 1964.

13. Jørgensen, M., S. Molbeck, and S. H. Johansen. Effect of decamethonium on head lift, hand grip, and respiratory muscle power in man. J. Appl. Physiol. 21: 509-512, 1966.

14. Leonard, B., J. H. Mitchell, M. Mizuno, N. Rube, B. Saltin, and N. H. Secher. Partial neuromuscular blockade and cardiovascular responses to static exercise in man. J. Physiol. Lond. 359: $365-379,1985$.

15. Mizuno, M. Humau skeletal muscle adaptation to training: with special reference to metabolic characteristics of slow- and fasttwitch fibres. J. Exerc. Sci. 2: 40-45, 1992.

16. Mizuno, M., L. O. Justesen, J. Bedolla, D. B. Friedman, N. H. Sccher, and B. Quistorff. Partial eurarization abolishes splitting of the inorganic phosphate peak in $1 \mathrm{P}$ P-NMR spectroscopy during intense forearm muscles in man. Acta Physiol. Scand. 139: 611-612, 1990

17. Mizuno, M., N. H. Secher, and B. Quistorff. ${ }^{31}$ P-NMR spectroscopy, rsEMG, and histochemical fiber types of human wrist flexor muscles. J. Appl. Physiol. 76: 531-538, 1994.

18. Molbech, S., and S. H. Johansen. Endurance time in static work during partial curarization. J. Appl. Physiol. 27: 44-48, 1969.

19. Ogata, T., T. Honda, and T. Seito. An electron microscopic study of differences in the fine structures of motor endplate in red, white, and intermediate muscle fibers of rat intercostal muscles. Acta Med. Olayama 21: 327-338, 1967.

20. Park, J. H., R. L. Brown, C. R. Park, K. McCully, M. Cohn, J. Haselgrove, and B. Chanee. Functional pools of oxidative and glycolytic fibers in human muscle observed by ${ }^{31} \mathrm{P}$ magnetic resonance spectroscopy during exercise. Proc. Natl. Acad. Sci. USA 84: 8976-8980, 1987.

21. Patton, W. D. M., and E. J. Zaimis. The action of $d$ tubocurarine and of decamethonium on respiratory and other muscles in the cat. J. Physiol. Lond. 112: 311-331, 1951.

22. Saltin, B., and P. D. Gollnick. Skeletal muscle adaptability significance for metabolism and performance. In: Handbooh of Physiology. Skeletal Muscle. Bethesda, MD: Am. Physiol. Soc., 1983, sect. 10, chapt. 19, p. 555-631.

23. Secher, N. H. Central nervous influence on fatigue. In: Endurance in Sport, edited by R. J. Shephard and P.-O. Âstrand. Oxford, UK: Blackwell, 1992, p. 96-106.

24. Secher, N. H., M. Mizuno, and B. Saltin. Adaptation of skeletal muscle to training. Bull. Eur. Physiopathol. Respir. 20. $453-457,1984$

25. Seeher, N. H., S. Rørsgaard, and O. Secher. Contralateral influence on recruitment of curarized muscle fibres during maximal voluntary extension of the legs. Acta Physiol. Scand. 103: $456-462,1978$

26. Secher, N. H., N. Rube, and O. Seeher. Effect of tubocurarine on human soleus and gastrocnemius muscles. Acta Anaesthesiol. Scand. 26: 231-234, 1982.

27. Secher, N. H., N. Rube, and O. Secher. Effect of tubocurarine and decamethonium on voluntary contractions in man. Acto Anaesthesiol. Scand. 27: 480-483, 1983.

28. Siegel, S. Nonparametric Statistics for the Behavioral. Sciences. Tokyo: McGraw-Hill, 1956.

29. Sterz, R., M. Pagala, and K. Peper, Postjunctional characteris tics of the endplates in mammalian fast and slow muscles. Pfluegers Arch. 398: 48-54, 1983.

30. 'T'aylor, D. J., P. J. Bore, P. Styles, D. G. Gadian, and G. K. Radda. Bioenergetics of intact human muscle. A ${ }^{31} \mathrm{P}$ nuclear magnetic resonance study. Mol. Biol. Med. 1: 77-94, 1983.

31. Vandenborne, K., K. MeCully, H. Kakihira, M. Prammer, L. Bolinger, J. A. Detre, K. De Meirleir, G. Walter, B. Chance, and J. S. Leigh. Metabolic heterogeneity in human calf muscle during maximal exercise. Proc. Natl. Acad. Sci. USA 88: 5714$5718,1991$.

32. Zaimis, E. Motor end-plate differences as a determining factor in the mode of action of neuromuscular blocking substances. $J$. Physiol. Lond. 122: 238-251, 1953. 